\title{
Effect of the Synaptic Plasma Membrane on the Stability of the Amyloid Precursor Protein Homodimer
}

\author{
Martina Audagnotto ${ }^{(1,2)}$, Thomas Lemmin ${ }^{(3)}$, Alessandro Barducci ${ }^{(4)(5)}$, Matteo Dal \\ $\operatorname{Peraro}^{(1,2)^{*}}$ \\ (1) Institute of Bioengineering, School of Life Sciences, École Polytechnique Fédérale de Lausanne \\ (EPFL), \\ (2) Swiss Institute of Bioinformatics (SIB), Lausanne, Switzerland \\ (3) Department of Pharmaceutical Chemistry, University of California San Francisco, San Francisco, \\ California, USA \\ (4) Inserm, U1054, Montpellier, France \\ (5) Université de Montpellier, CNRS, UMR 5048, Centre de Biochimie Structurale, Montpellier, France
}

\section{Computational Material and Methods}

Atomistic MD simulations. The initial dimer conformation of APP-TM is taken from experimental NMR structure of APP-TM $\mathrm{G}_{709} \mathrm{XXXA}_{713}$ in zwitterionic dodecylphosphocholine (DPC) micelles (PDB: 2LZ3) ${ }^{1}$. All the membrane systems (young-SPM, old-SPM, POPC, POPC/PAPC) were generated using LipidBuilder (http://lipidbuilder.epfl.ch) ${ }^{2}$, modeled using the CHARMM36 force field ${ }^{3-4}$ and solvated with the TIP3P water model $\mathrm{l}^{5}$ and equilibrated following the protocol reported in ref. 2. The distribution of the lipids and cholesterol between the leaflets is summarized in Table S1. The NMR structure (PDB: 2LZ3) was inserted in a pre-equilibrated membrane (of $80 \times 80 \AA^{2}$ size) and any clashes were removed. 5 independent replicas were run for young- and old-SPM systems as well as the three control bilayers featuring (i) SPM without cholesterol, (ii) POPC and (iii) POPC/PAPC mixture (Table S1). We run 200 ns MD production for each replica of SPM (young, old and without cholesterol) and for POPC/PAPC system, while we extend up to $300 \mathrm{~ns}$ all the replicas of POPC for a total of $5.5 \mu$ s aggregate MD time. All atomistic MD simulations were performed using NAMD 2.9 $9^{3}$ using the CHARMM36 force field. The periodic electrostatic interactions were computed using particle mesh Ewald (PME) summation with grid spacing smaller than $1 \AA$. Constant pressure of $1 \mathrm{~atm}$ was maintained with Langevin piston dynamics, a $200-\mathrm{fs}$ decay period, and 50 -fs time constant. Constant physiological temperature $(310 \mathrm{~K})$ was imposed by using Langevin dynamics ${ }^{7}$ with a damping coefficient of $1.0 \mathrm{ps}$. All the analysis were performed using as time interval $2 \mathrm{ps}$.

Coarse-Grained (CG) MD simulations. The CG simulations are based on DAFT protocol ${ }^{8}$ where the helical models are coarse-grained using martinize $e^{9}$ and aligned along the $\mathrm{z}$-axis using PYMOL. 500 starting structures, where the monomers are separated by $35 \AA$, were generated and the outcome configurations were processed with insane $e^{10}$, which generate the realistic SPM membrane and solvent. Each system was minimized and equilibrated for 10 ps in NVT MD using 2 fs of time step. Afterward 100 ps NpT MD with a time step of $20 \mathrm{fs}$ was applied in order to bring the temperature and pressure in a range of $310 \mathrm{~K}$ and 1 bar respectively. The temperature was controlled using the Bussi thermostat with a coupling time of $1 \mathrm{ps}^{11}$ while the pressure was controlled by weak semi-isotropic coupling with a reference pressure of 1 bar and a compressibility of $3 \times 10^{-4} \mathrm{bar}^{-1}$. The production simulations were performed for $512 \mathrm{~ns}$ with a time step of $20 \mathrm{fs}$. All the simulations were performed using GROMACS 4.5.6 ${ }^{12}$ and the dimerization and relaxation properties were analyzed computing the non-bonded interaction energy over time between the two monomers embedded in the SPM (see results in Figure S1). 


\section{PCA clustering analysis:}

Principal component analysis (PCA) was performed on roughly 44000 frames of MD trajectories. For the PCA, only $\mathrm{C} \alpha$ beads coordinate were considered. Eigenvectors describing at least $80 \%$ of the protein $\mathrm{C} \alpha$ fluctuation were selected to describe the protein flexibility. Following the projection of trajectory frames on relevant eigenvectors, a hierarchical clustering scheme ${ }^{13}$ was applied in order to extract representative protein conformations.

APP-TM/lipid interactions. The interaction between lipids and APP-TM dimer was characterized by computing the distance between the center of mass of the atoms involved in the two dimerization motifs $\left(\mathrm{G}_{700} \mathrm{XXXG}_{704} \mathrm{XXXG}_{708}\right.$ and $\left.\mathrm{G}_{709} \mathrm{XXXA}_{713}\right)$ and the center of mass of the atoms that form the unsaturation in the lipids.

APP-TM/cholesterol interactions. The interaction between cholesterol molecules and APP-TM dimer was characterized by computing the (i) angle $(\theta)$ between the principal axis of APP-TM dimer and the principal axis of the cholesterol molecules (i.e. aromatic body in the case of the $\mathrm{G}_{700} \mathrm{XXXG}_{704} \mathrm{XXXG}_{708}$ motif and hydrocarbon tail in the case of $\mathrm{G}_{709} \mathrm{XXXA}_{713}$ ) and (ii) the distance between the center of mass of all the atoms involved in the two dimerization motifs $\left(\mathrm{G}_{700} \mathrm{XXXG}_{704} \mathrm{XXXG}_{708}\right.$ and $\left.\mathrm{G}_{709} \mathrm{XXXA}_{713}\right)$ and the center of mass of the cholesterol molecules (see illustration below).

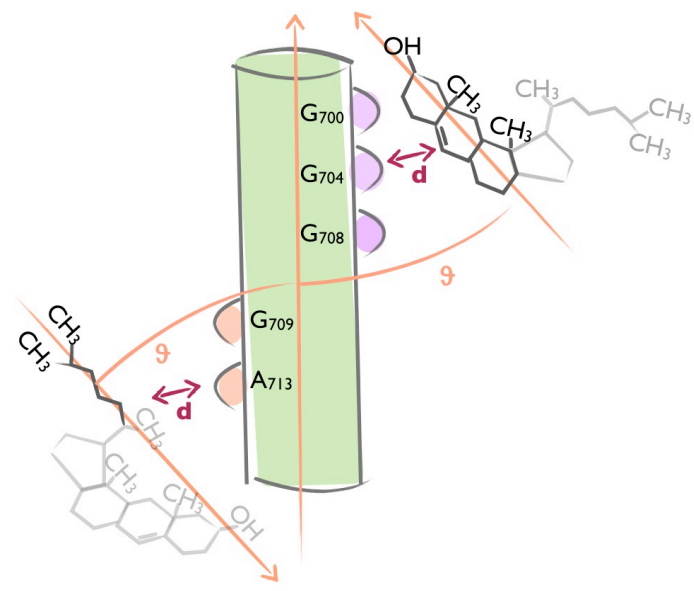

Scheme 1. Geometrical characterization of cholesterol/APP-TM interactions. 
Table S1. Lipids distribution of the Synaptic Plasma Membrane models. The relative abundance of the different lipids species is given for both leaflets.

\begin{tabular}{|c|c|c|c|c|}
\hline & \multicolumn{2}{|c|}{ young-SPM } & \multicolumn{2}{|c|}{ old-SPM } \\
\hline & $\%$ inner & $\%$ outer & $\%$ inner & $\%$ outer \\
\hline POPC (16:0/18:1) & $4(14)$ & $11(8)$ & $5(12)$ & $8(9)$ \\
\hline PAPC (16:0/20:4) & $4(13)$ & $10(8)$ & $5(10)$ & $7(9)$ \\
\hline HDPC (16:0/22:6) & $4(14)$ & $11(8)$ & $5(12)$ & $8(8)$ \\
\hline SOPC (18:0/18:1) & $6(16)$ & $13(12)$ & $6(15)$ & $10(11)$ \\
\hline POPE (16:0/18:1) & $4(6)$ & $9(7)$ & $4(9)$ & $7(6)$ \\
\hline SAPE (18:0/20:4) & $2(6)$ & $5(4)$ & $3(6)$ & $4(6)$ \\
\hline SDPE (18:0/22:6) & $5(14)$ & $11(6)$ & $5(11)$ & $9(8)$ \\
\hline ODPS (18:0/22:6) & $5(7)$ & $6(9)$ & $6(6)$ & $5(10)$ \\
\hline SOPS (18:0/18:1) & $1(3)$ & $2(2)$ & $2(2)$ & $1(4)$ \\
\hline CHOL & $64(28)$ & $23(133)$ & $59(62)$ & $41(111)$ \\
\hline
\end{tabular}

Table S2. Lipids distribution of the control membrane models. The relative abundance of the different lipids species is given for both leaflets (in parenthesis the number of lipid molecules included)

\begin{tabular}{|c|c|c|c|c|c|c|}
\hline & \multicolumn{2}{|c|}{ no-cholesterol SPM } & \multicolumn{2}{|c|}{ POPC } & \multicolumn{2}{|c|}{ POPC/PAPC } \\
\hline & \%inner & \%outer & \%inner & \%outer & \%inner & \%outer \\
\hline $\begin{array}{l}\text { POPC } \\
(16: 0 / 18: 1)\end{array}$ & $12(21)$ & $14(17)$ & $100(224)$ & $100(224)$ & $50(112)$ & $50(112)$ \\
\hline $\begin{array}{l}\text { PAPC } \\
(16: 0 / 20: 4)\end{array}$ & $15(18)$ & $17(15)$ & & & $50(112)$ & $50(112)$ \\
\hline $\begin{array}{l}\text { HDPC } \\
(16: 0 / 22: 6)\end{array}$ & $12(17)$ & $14(16)$ & & & & \\
\hline $\begin{array}{l}\text { SOPC } \\
(18: 0 / 18: 1)\end{array}$ & $15(25)$ & $17(21)$ & & & & \\
\hline $\begin{array}{l}\text { POPE } \\
(16: 0 / 18: 1)\end{array}$ & $10(13)$ & $11(12)$ & & & & \\
\hline SAPE (18:0/20:4) & $6(10)$ & $7(8)$ & & & & \\
\hline SDPE (18:0/22:6) & $13(21)$ & $15(18)$ & & & & \\
\hline $\begin{array}{l}\text { ODPS } \\
(18: 0 / 22: 6)\end{array}$ & $15(12)$ & $8(17)$ & & & & \\
\hline SOPS (18:0/18:1) & $4(3)$ & $2(8)$ & & & & \\
\hline CHOL & -- & -- & & & & \\
\hline
\end{tabular}



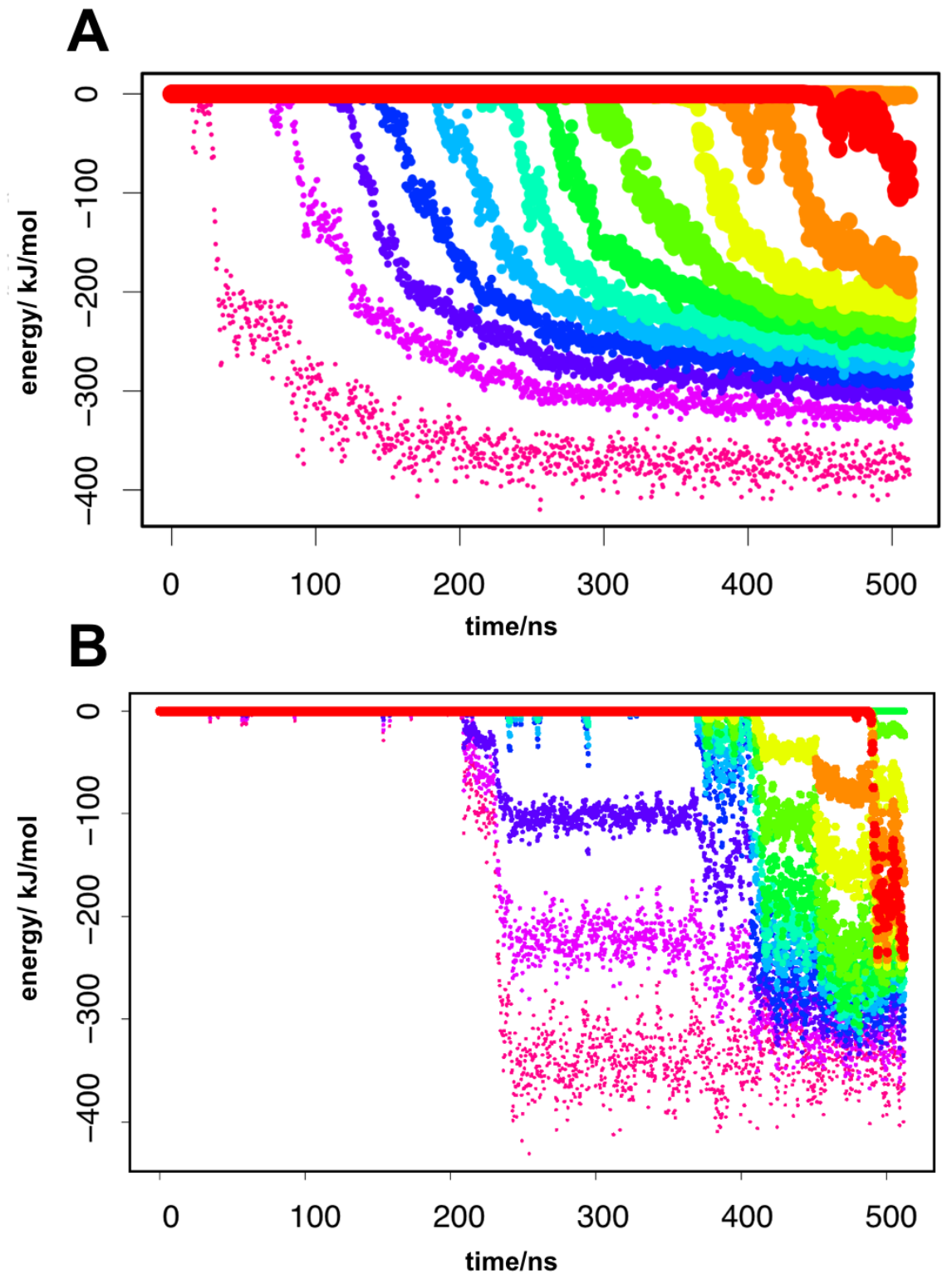

Figure S1. DAFT interaction energy between monomers during the MD time evolution. The graphs show the time evolution of the non-bonded interaction energy of the APP-TM monomers in (A) SPM and (B) POPC bilayer. At each time, the interaction energy is characterized by marking the $5 \%$ points (i.e., vigintile). In the case of SPM and POPC we observed that 11 out of 21 vigintile bands are below zero, indicating that in both systems $52 \%$ of the total simulations represent an interaction between the two monomers. 


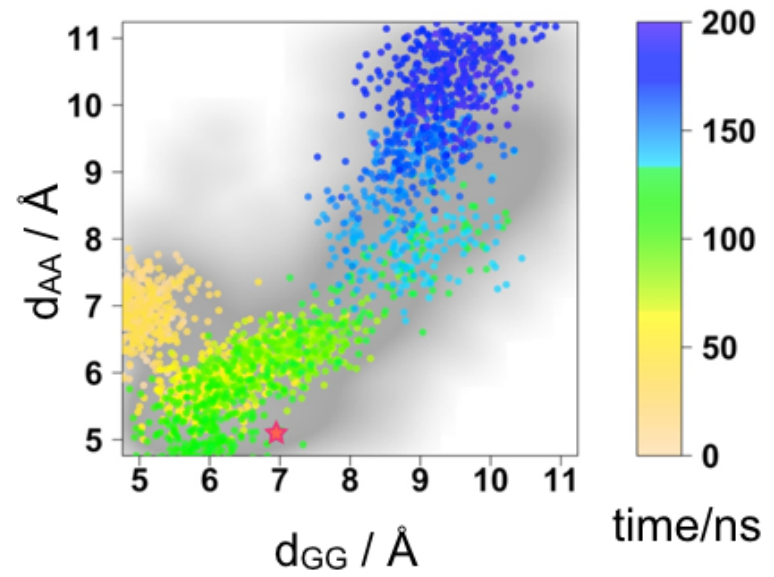

Figure S2. APP-TM dimer conformation in SPM without cholesterol. The color scale shows the time evolution. The grey surface represents the averaged conformational space explored by the dimer at different membrane lipids compositions in all the replicas. The red star is the value of $\mathrm{d}_{\mathrm{GG}}$ and $\mathrm{d}_{\mathrm{AA}}$ from the experimentally derived structure of APP-TM G $\mathrm{G}_{709} \mathrm{XXXA}_{713}$ dimer (PDB: 2LZ3) ${ }^{1}$. Note that the APP-TM in SPM without cholesterol explored the monomeric conformation characterized by $\mathrm{d}_{\mathrm{GG}} \approx 10 \AA$ and $\mathrm{d}_{\mathrm{AA}} \approx 11 \AA$ 

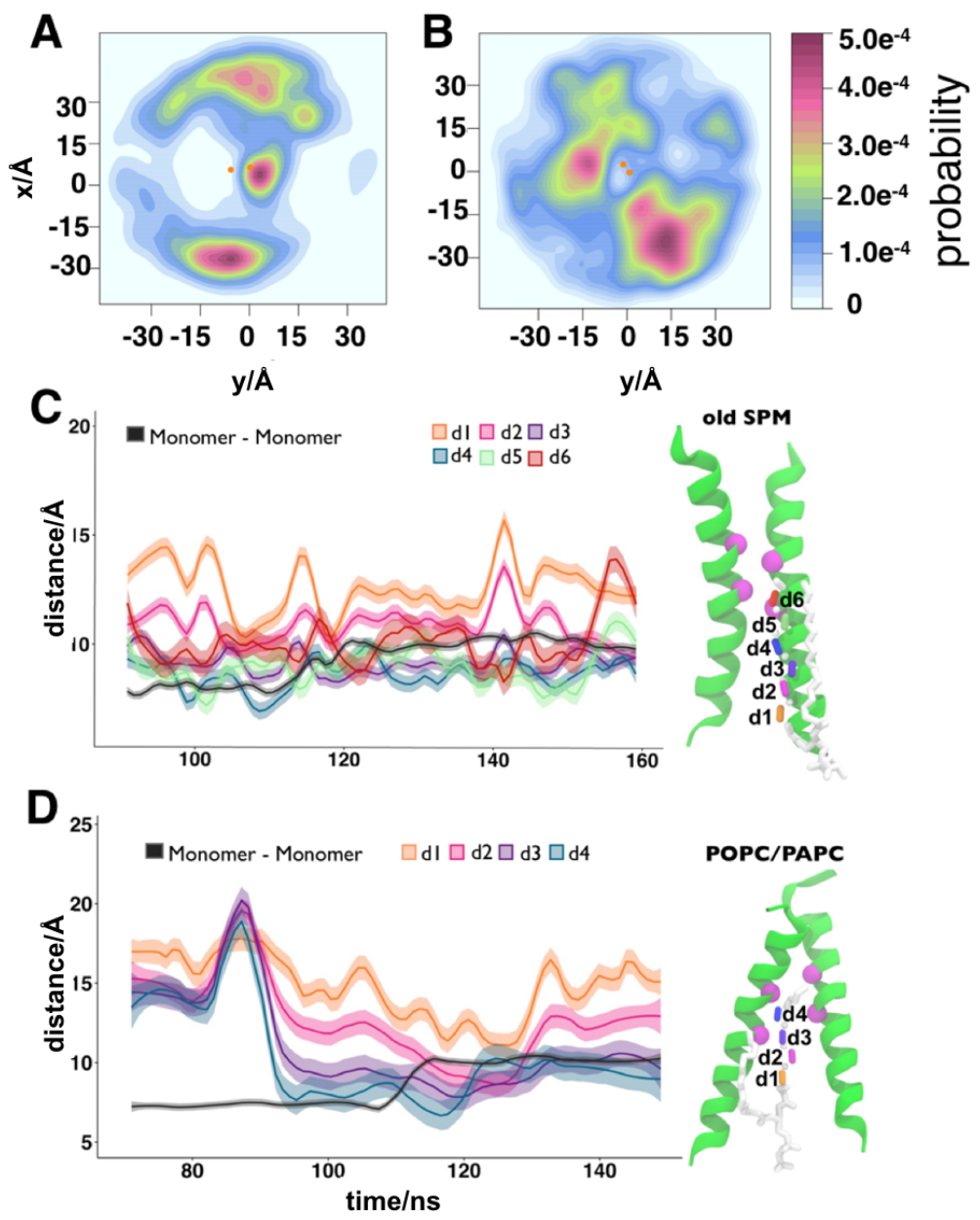

Figure S3. Effect of unsaturated lipids on dimer stability. Most representative high unsaturated lipid distribution for (A) $\mathrm{G}_{709} \mathrm{XXXA}_{713}$ and (B) $\mathrm{G}_{700} \mathrm{XXXG}_{704} \mathrm{XXXG}_{708}$ embedded in the complex SPM. Despite the presence of high unsaturated lipids close to the $\mathrm{G}_{700} \mathrm{XXXG}_{704} \mathrm{XXXG}_{708}$ motif, the dimer is stable in all the replica. The orange dots represent the averaged position of the helix in all the simulated replicas. Lipid-protein interactions in function of time for the old-SPM bilayer (C) and the POPC/PAPC bilayer (D). The black line is the distance between the APP-TM monomers averaged over time. Distance between each lipid unsaturation and the $\mathrm{G}_{709} \mathrm{XXXA}_{713}$ motif residues is colored by the unsaturation position. 

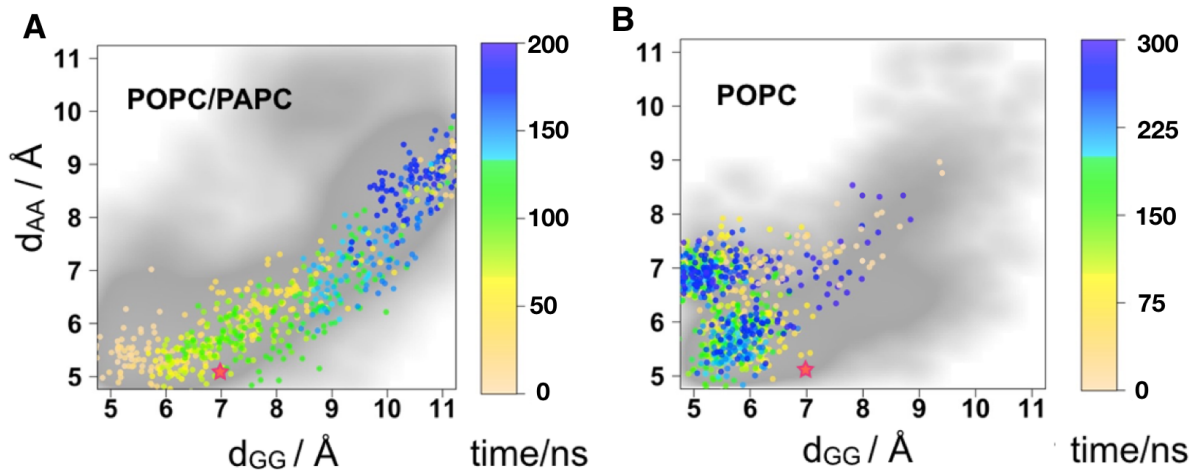

Figure S4. The $\mathbf{G}_{709} \mathbf{X X X A}_{713}$ dimer is not stable in POPC/PAPC membrane. APP-TM dimer evolution during MD in different membrane models: (A) POPC/PAPC and (B) POPC. The grey surface represents the global conformational space explored by each system, while the red star indicates the initial NMR values of $\mathrm{d}_{\mathrm{GG}}$ and $\mathrm{d}_{\mathrm{AA}}$. 

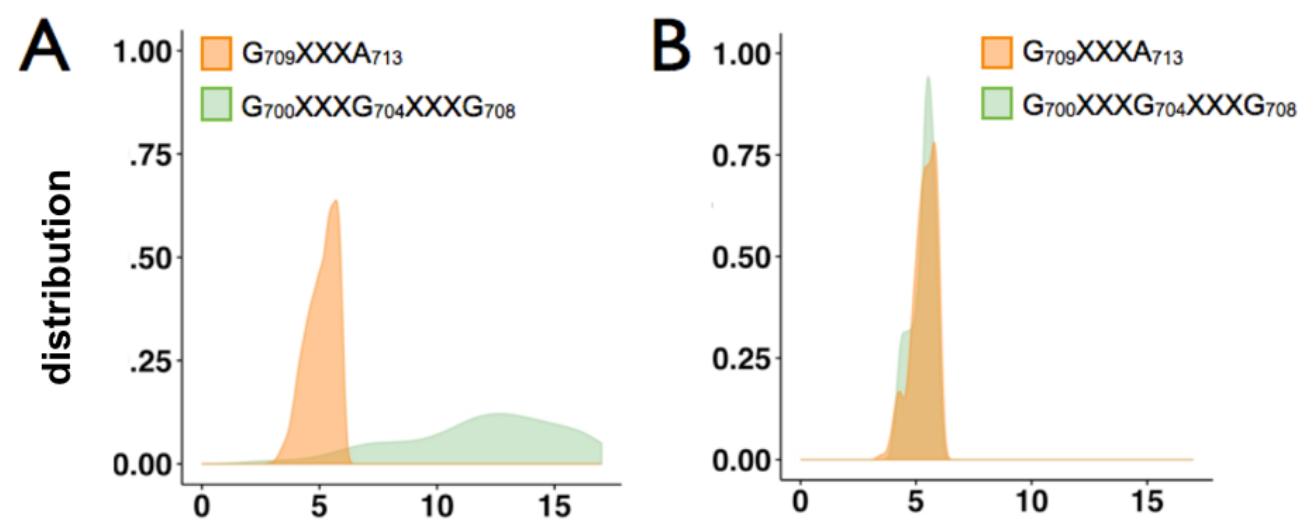

Distance from dimer interface/A

Distance from dimer interface/A

Figure S5. Distribution of cholesterol around the $G_{709} X X X A_{713 \text { and }} G_{700} X X X G_{704} X X X G_{708}$ motifs. Cholesterol distribution around the $\mathrm{G}_{700} \mathrm{XXXG}_{704} \mathrm{XXXG}_{708}$ APP-TM dimer (A) and $\mathrm{G}_{709} \mathrm{XXXA}_{713}$ dimer (B), which then dissociate during MD simulations. In the case of $\mathrm{G}_{700} \mathrm{XXXG}_{704} \mathrm{XXXG}_{708}$ dimer we observed that cholesterol has a strong preference for $\mathrm{G}_{709} \mathrm{XXXA}_{713}$ since the $\mathrm{G}_{700} \mathrm{XXXG}_{704} \mathrm{XXXG}_{708}$ motif is involved in dimerization. In the case of $\mathrm{G}_{709} \mathrm{XXXA}_{713}$ dimer MD simulations showed that the dimer is not stable and cholesterol showed an indiscriminate preference for both $\mathrm{G}_{700} \mathrm{XXXG}_{704} \mathrm{XXXG}_{708}$ and $\mathrm{G}_{709} \mathrm{XXXA}_{713}$ motifs. 


\section{REFERENCE}

1. Chen, W.; Gamache, E.; Rosenman, D. J.; Xie, J.; Lopez, M. M.; Li, Y. M.; Wang, C., Familial Alzheimer's Mutations within Apptm Increase Abeta42 Production by Enhancing Accessibility of Epsilon-Cleavage Site. Nat. Commun. 2014, 5, 3037.

2. Bovigny, C.; Tamo, G.; Lemmin, T.; Maino, N.; Dal Peraro, M., Lipid Builder: A Framework to Build Realistic Models for Biological Membranes. J. Chem. Inf. Model 2015, 55, 2491-2499.

3. Klauda, J. B.; Venable, R. M.; Freites, J. A.; O'Connor, J. W.; Tobias, D. J.; Mondragon-Ramirez, C.; Vorobyov, I.; MacKerell, A. D., Jr.; Pastor, R. W., Update of the Charmm All-Atom Additive Force Field for Lipids: Validation on Six Lipid Types. J. Phys. Chem. B 2010, 114, 7830-7843.

4. Phillips, J. C.; Braun, R.; Wang, W.; Gumbart, J.; Tajkhorshid, E.; Villa, E.; Chipot, C.; Skeel, R. D.; Kale, L.; Schulten, K., Scalable Molecular Dynamics with NAMD. J. Comput. Chem. 2005, 26, 1781-1802.

5. Jorgensen, W. L.; Chandrasekhar, J.; Madura, J. D.; Impey, R. W.; Klein, M. L., Comparison of Simple Potential Functions for Simulating Liquid Water. J. Chem. Phys. 1983, 79, 926-935.

6. Feller, S. E.; Zhang, Y. H.; Pastor, R. W.; Brooks, B. R., Constant-Pressure Molecular-Dynamics Simulation - the Langevin Piston Method. J. Chem. Phys. 1995, 103, 4613-4621.

7. Brunger, A.; Brooks, C. L.; Karplus, M., Stochastic Boundary-Conditions for Molecular-Dynamics Simulations of St2 Water. Chem. Phys. Lett. 1984, 105, 495-500.

8. Wassenaar, T. A.; Pluhackova, K.; Moussatova, A.; Sengupta, D.; Marrink, S. J.; Tieleman, D. P.; Bockmann, R. A., High-Throughput Simulations of Dimer and Trimer Assembly of Membrane Proteins. The Daft Approach. J. Chem. Theory Comput. 2015, 11, 2278-2291.

9. de Jong, D. H.; Singh, G.; Bennett, W. F. D.; Arnarez, C.; Wassenaar, T. A.; Schafer, L. V.; Periole, X.; Tieleman, D. P.; Marrink, S. J., Improved Parameters for the Martini Coarse-Grained Protein Force Field. J. Chem. Theory Comput. 2013, 9, 687-697.

10. Wassenaar, T. A.; Ingolfsson, H. I.; Bockmann, R. A.; Tieleman, D. P.; Marrink, S. J., Computational Lipidomics with Insane: A Versatile Tool for Generating Custom Membranes for Molecular Simulations. J. Chem. Theory Comput. 2015, $11,2144-2155$.

11. Bussi, G.; Donadio, D.; Parrinello, M., Canonical Sampling through Velocity Rescaling. J. Chem. Phys. 2007, 126, 014101.

12. Hess, B.; Kutzner, C.; van der Spoel, D.; Lindahl, E., Gromacs 4: Algorithms for Highly Efficient, Load-Balanced, and Scalable Molecular Simulation. J. Chem. Theory Comput. 2008, 4, 435-447.

13. Lindahl, E.; Hess, B.; van der Spoel, D., Gromacs 3.0: A Package for Molecular Simulation and Trajectory Analysis. J. Mol. Model. 2001, 7, 306-317. 